\title{
Study on Correlation and Path Analysis for Yield and Yield Components in Cowpea [Vigna unguiculata (L.) Walp]
}

\author{
P. D. Waghmare*, U. B. Pethe and G. R. Sabale \\ Dr. Balasaheb Sawant Konkan Krishi Vidyapeeth, Dapoli (MS), India \\ *Corresponding author
}

Keywords

Cowpea,

Genotypes,

Correlation, Path

co-efficient analysis

Article Info

Accepted:

04 November 2019

Available Online:

10 December 2019

\section{A B S T R A C T}

The present experiment was carried out on correlation and path analysis in cowpea [Vigna unguiculata (L.) Walp.] during 2016-17. The study was consists 30 genotypes of cowpea using randomized block design with two replications. The phenotypic and genotypic correlation coefficient revealed that seed yield per plant was significantly and positively correlated with dry matter yield per plant (0.8502), harvest index (0.8082), 100 seed weight (0.6377), number of pods per plant $(0.4413)$ and number of seeds per pod (0.2815). However, plant height (-0.0022) showed significantly and negatively correlated with seed yield per plant. The character pod length $(-0.2377)$ showed negative non-significantly correlated with seed yield per plant. Path coefficient analysis of different yield and yield contributing traits on number of pods per plant, number of seeds per pod, pod length $(\mathrm{cm})$, hundred seed weight (g) and harvest index (\%) exhibited positive direct effects on seed yield per plant these characters play a major role in recombination breeding and suggested that direct selection based on these traits will be rewarded for crop improvement of cowpea.

\section{Introduction}

Pulses are the rich source of proteins, vitamins and minerals for the predominantly vegetarian population of our country. Cowpea [Vigna unguiculata (L.) Walp] is one of the most widely adapted, drought-tolerant, versatile and nutritious pulse crop. In the world, the total area of pulse production is $78 \mathrm{M}$ hectare with production of $70 \mathrm{MT}$ and productivity 908 $\mathrm{kg} / \mathrm{ha}$ (Anon., 2012). In India, the total area of pulses production is $72.3 \mathrm{M}$ hectare with production of $64.4 \mathrm{MT}$ and productivity 890 $\mathrm{kg} / \mathrm{ha}$, whereas Maharashtra contributes $12 \%$ of a total production of Cowpea. For any crop improvement program, evaluation of germplasm to assess the existing variability is the first step. Greater the variability present in the initial material better would be the chances for evolving desired types. A clear 
understanding of variability of various characters of the breeding materials is an asset to the plant breeder for selecting superior genotypes on the basis of their phenotypic expression. In this regards estimates of genotypic and phenotypic variance for various quantitative characters along with heritability and genetic advance expected by selection for yield and its components are useful in designing an effective breeding program.

\section{Materials and Methods}

The present investigation "Study on correlation and path analysis in cowpea [Vigna unguiculata (L.) Walp.] was carried out at the Educational \& Research Farm of Department of Agil. Botany, College of Agriculture, Dapoli, Maharashtra, India during Rabi, 2016. The study was under taken on 30 genotypes of cowpea using randomized block design with two replications. Keeping a plot size is of 2.0 $\mathrm{m} \times 1.5 \mathrm{~m}$. The plot was selected on the basis of suitability of the land for cultivation of cowpea. The data was recorded on following quantitative parameters plant height, days to first flowering, days to $50 \%$ flowering, days to maturity number primary branches per plant, number of pods for plant, pod length, number of seeds per pod, hundred seed weight and harvest index. The analysis was done as per Panse and Sukhatme (1985), Burton and De Vane (1953), Johnson et al., (1955), Dewey and Lu (1959).

\section{Results and Discussion}

Correlation studies In order to find out the association between yield and yield contributing characters, the genotypic and phenotypic correlation coefficients were estimated and presented in Table 1.

\section{Phenotypic and genotypic correlation coefficient}

The result on phenotypic and genotypic correlation coefficient revealed that seed yield per plant was significantly and positively correlated with dry matter yield per plant (0.8502), harvest index (0.8082), hundred seed weight (0.6377), number of pods per plant (0.4413) and number of seeds per pod (0.2815). However, plant height (-0.0022) showed significantly and negatively correlated with seed yield per plant. The character pod length (-0.2377) showed negative non significantly correlated with seed yield per plant. These results are in similar with the finding of Singh et al., (2004). Number of pods per plant showed positive significant correlation with number of primary branches per plant (0.8842), number of pods per plant (0.8371), negative significant correlation with days to $50 \%$ flowering (-0.0043), pod length (-0.1467), negative, significant correlation with 100 seed weight $(-0.2635)$. These results are in consonance with the finding of Malarvizhi and Rangasamy (2003) and Oseni et al., (1992) in case of pods per plant. Number of pods per plant showed positive correlation with harvest index (0.5010). It had positive non-significant correlation with dry matter yield per plant (0.2522), pod length (0.0701) and hundred seed weight (0.0118). The same result was recorded by Patel et al., (2016) with pod length in cowpea. Pod length is an important parameter considered for improvement which was positively correlated with harvest index (0.2370), dry matter yield per plant (0.1724) and number of seeds per pod (0.1491). Similar results were observed by Sapra and Javia (2014) for pod yield at genotypic and phenotypic level, but negatively correlated for hundred seed weight (Table 2).

\section{Path Co-efficient analyses}

It was analyzed for yield and yield contributing traits are presented in (Table 3 and 4). It was observed that genotypic direct and indirect effects were higher than their corresponding phenotypic values. 
Table.1 Estimates of phenotypic correlation coefficient for 12 quantitative characters

\begin{tabular}{|c|c|c|c|c|c|c|c|c|c|c|c|c|}
\hline Character & $\begin{array}{c}\text { Days to } \\
\text { first } \\
\text { flowering }\end{array}$ & $\begin{array}{c}\text { Days to } \\
50 \% \\
\text { flowering }\end{array}$ & $\begin{array}{l}\text { Days to } \\
\text { maturity }\end{array}$ & $\begin{array}{c}\text { Plant } \\
\text { height } \\
(\mathbf{c m})\end{array}$ & $\begin{array}{c}\text { Number } \\
\text { of } \\
\text { primary } \\
\text { branches } \\
\text { per plant }\end{array}$ & $\begin{array}{c}\text { Number } \\
\text { of pods } \\
\text { per } \\
\text { plant }\end{array}$ & $\begin{array}{c}\text { Pod } \\
\text { length } \\
(\mathbf{c m})\end{array}$ & $\begin{array}{c}\text { Number } \\
\text { of seed } \\
\text { per pod }\end{array}$ & $\begin{array}{l}\text { Dry matter } \\
\text { yield per } \\
\text { plant (g) }\end{array}$ & $\begin{array}{l}\text { Hundred } \\
\text { seed } \\
\text { weight (g) }\end{array}$ & $\begin{array}{c}\text { Harvest } \\
\text { index } \\
(\%)\end{array}$ & $\begin{array}{c}\text { Seed } \\
\text { yield per } \\
\text { plant }(\mathrm{g})\end{array}$ \\
\hline $\begin{array}{l}\text { Days to first } \\
\text { flowering }\end{array}$ & 1.0000 & $0.8872 * *$ & $0.8189 * *$ & 0.2179 & 0.1336 & $0.3859 *$ & 0.2198 & -0.0354 & 0.0496 & -0.0245 & 0.2294 & 0.1568 \\
\hline $\begin{array}{c}\text { Days to } 50 \% \\
\text { flowering }\end{array}$ & & 1.0000 & $0.8905^{* *}$ & 0.1846 & 0.1165 & $0.4088 * *$ & $0.3028 *$ & -0.0264 & 0.1154 & -0.0196 & 0.2111 & 0.1880 \\
\hline Days to maturity & & & 1.0000 & 0.2228 & 0.0711 & $0.2566^{*}$ & $0.2961 *$ & 0.0111 & 0.0885 & 0.0232 & 0.1776 & 0.1476 \\
\hline $\begin{array}{c}\text { Plant height } \\
(\mathbf{c m})\end{array}$ & & & & 1.0000 & 0.0737 & 0.1504 & 0.1666 & -0.0715 & 0.0463 & -0.1229 & -0.0448 & -0.0022 \\
\hline $\begin{array}{c}\text { Number of } \\
\text { primary } \\
\text { branches per } \\
\text { plant }\end{array}$ & & & & & 1.0000 & $0.3389 *$ & 0.0448 & -0.0414 & 0.1134 & 0.0252 & 0.1284 & 0.1357 \\
\hline $\begin{array}{c}\text { Number of pods } \\
\text { per plant }\end{array}$ & & & & & & 1.0000 & 0.0701 & $\stackrel{-}{-}$ & 0.2522 & 0.0118 & $0.5010 * *$ & $0.4413 * *$ \\
\hline Pod length $(\mathrm{cm})$ & & & & & & & 1.0000 & 0.1491 & 0.1724 & 0.0800 & 0.2370 & 0.2377 \\
\hline $\begin{array}{l}\text { Number of seed } \\
\text { per pod }\end{array}$ & & & & & & & & 1.0000 & $0.5960 * *$ & 0.0593 & 0.0746 & $0.4103 * *$ \\
\hline $\begin{array}{l}\text { Dry matter yield } \\
\text { per plant (g) }\end{array}$ & & & & & & & & & 1.0000 & $0.5267 * *$ & $0.3836^{*}$ & $0.8502 * *$ \\
\hline $\begin{array}{l}\text { Hundred seed } \\
\text { weight (g) }\end{array}$ & & & & & & & & & & 1.0000 & $0.5021 * *$ & $0.6377 * *$ \\
\hline $\begin{array}{c}\text { Harvest index } \\
(\%)\end{array}$ & & & & & & & & & & & 1.0000 & $0.8082 * *$ \\
\hline $\begin{array}{l}\text { Seed yield per } \\
\text { plant }(\mathrm{g})\end{array}$ & & & & & & & & & & & & 1.0000 \\
\hline
\end{tabular}


Table.2 Estimates of genotypic correlation coefficient for 12 quantitative characters

\begin{tabular}{|c|c|c|c|c|c|c|c|c|c|c|c|c|}
\hline Character & $\begin{array}{c}\text { Days to } \\
\text { first } \\
\text { flowering }\end{array}$ & $\begin{array}{c}\text { Days to } \\
50 \% \\
\text { flowering }\end{array}$ & $\begin{array}{l}\text { Days to } \\
\text { maturity }\end{array}$ & $\begin{array}{c}\text { Plant } \\
\text { height } \\
\text { (cm) }\end{array}$ & $\begin{array}{l}\text { Number } \\
\text { of } \\
\text { primary } \\
\text { branches } \\
\text { per plant }\end{array}$ & $\begin{array}{l}\text { Number } \\
\text { of pods } \\
\text { per plant }\end{array}$ & $\begin{array}{c}\text { Pod } \\
\text { length } \\
\text { (cm) }\end{array}$ & $\begin{array}{c}\text { Number } \\
\text { of seed } \\
\text { per pod }\end{array}$ & $\begin{array}{c}\text { Dry } \\
\text { matter } \\
\text { yield per } \\
\text { plant (g) }\end{array}$ & $\begin{array}{l}\text { Hundred } \\
\text { seed } \\
\text { weight (g) }\end{array}$ & $\begin{array}{c}\text { Harvest } \\
\text { index } \\
(\%)\end{array}$ & $\begin{array}{c}\text { Seed yield } \\
\text { per plant } \\
\text { (g) }\end{array}$ \\
\hline $\begin{array}{l}\text { Days to first } \\
\text { flowering }\end{array}$ & 1.0000 & $0.9883 * *$ & 0.9801 & 0.2677 & 0.2025 & $0.4028 * *$ & 0.2331 & -0.0509 & 0.0571 & -0.0215 & $0.3266 *$ & 0.1811 \\
\hline $\begin{array}{c}\text { Days to } 50 \% \\
\text { flowering }\end{array}$ & & 1.0000 & 0.9935 & 0.2262 & 0.1584 & $0.4298 * *$ & $0.3231 *$ & -0.0323 & 0.1410 & -0.0250 & $0.3479 *$ & 0.2412 \\
\hline Days to maturity & & & 1.0000 & $0.3711 *$ & -0.0208 & $0.3091 *$ & $0.4182 * *$ & -0.0163 & 0.1883 & 0.0406 & 0.2391 & 0.2263 \\
\hline Plant height (cm) & & & & 1.0000 & 0.1898 & 0.1612 & 0.1166 & -0.1043 & 0.0690 & -0.1533 & 0.0167 & 0.0324 \\
\hline $\begin{array}{l}\text { Number of primary } \\
\text { branches per plant }\end{array}$ & & & & & 1.0000 & $0.4319 * *$ & 0.1363 & -0.0699 & 0.1797 & 0.0244 & $0.3645^{*}$ & $0.2734^{*}$ \\
\hline $\begin{array}{c}\text { Number of pods per } \\
\text { plant }\end{array}$ & & & & & & 1.0000 & 0.0664 & -0.3139 & $0.2798^{*}$ & 0.0125 & $0.6403 * *$ & $0.4680 * *$ \\
\hline Pod length (cm) & & & & & & & 1.0000 & 0.1566 & 0.1875 & 0.0874 & 0.2497 & 0.2261 \\
\hline $\begin{array}{l}\text { Number of seed per } \\
\text { pod }\end{array}$ & & & & & & & & 1.0000 & 0.6264 & 0.0584 & 0.1080 & $0.4346 * *$ \\
\hline $\begin{array}{c}\text { Dry matter yield per } \\
\text { plant (g) }\end{array}$ & & & & & & & & & 1.0000 & 0.5547 & $0.6721 * *$ & $0.9267 * *$ \\
\hline $\begin{array}{c}\text { Hundred seed } \\
\text { weight }(\mathrm{g})\end{array}$ & & & & & & & & & & 1.0000 & $0.6293 * *$ & $0.6552 * *$ \\
\hline Harvest index (\%) & & & & & & & & & & & 1.0000 & $0.9003 * *$ \\
\hline $\begin{array}{l}\text { Seed yield per plant } \\
\text { (g) }\end{array}$ & & & & & & & & & & & & 1.0000 \\
\hline
\end{tabular}


Table.3 Path analysis at phenotypic level for 12 quantitative characters

\begin{tabular}{|c|c|c|c|c|c|c|c|c|c|c|c|c|}
\hline Character & $\begin{array}{c}\text { Days to } \\
\text { first } \\
\text { flowering }\end{array}$ & $\begin{array}{c}\text { Days to } \\
50 \% \\
\text { flowering }\end{array}$ & $\begin{array}{l}\text { Days to } \\
\text { maturity }\end{array}$ & $\begin{array}{c}\text { Plant } \\
\text { height } \\
\text { (cm) }\end{array}$ & $\begin{array}{c}\text { Number } \\
\text { of } \\
\text { primary } \\
\text { branches } \\
\text { per } \\
\text { plant }\end{array}$ & $\begin{array}{l}\text { Number } \\
\text { of pods } \\
\text { per } \\
\text { plant }\end{array}$ & $\begin{array}{c}\text { Pod } \\
\text { length } \\
\text { (cm) }\end{array}$ & $\begin{array}{l}\text { Number } \\
\text { of seed } \\
\text { per pod }\end{array}$ & $\begin{array}{c}\text { Dry } \\
\text { matter } \\
\text { yield } \\
\text { per } \\
\text { plant } \\
\text { (g) }\end{array}$ & $\begin{array}{l}\text { Hundred } \\
\text { seed } \\
\text { weight } \\
\text { (g) }\end{array}$ & $\begin{array}{c}\text { Harvest } \\
\text { index } \\
(\%)\end{array}$ & $\begin{array}{l}\text { Seed } \\
\text { yield } \\
\text { per } \\
\text { plant } \\
(\mathrm{g})\end{array}$ \\
\hline $\begin{array}{l}\text { Days to first } \\
\text { flowering }\end{array}$ & -0.0025 & 0.0198 & -0.0231 & 0.0005 & -0.0017 & 0.0151 & 0.0000 & -0.0012 & 0.0290 & -0.0015 & 0.1225 & 0.1568 \\
\hline $\begin{array}{l}\text { Days to } 50 \% \\
\text { flowering }\end{array}$ & -0.0022 & 0.0223 & -0.0251 & 0.0004 & -0.0015 & 0.0160 & 0.0000 & -0.0009 & 0.0675 & -0.0012 & 0.1127 & 0.1880 \\
\hline $\begin{array}{c}\text { Days to } \\
\text { maturity }\end{array}$ & -0.0021 & 0.0199 & -0.0282 & 0.0005 & -0.0009 & 0.0100 & 0.0000 & -0.0024 & 0.0271 & -0.0075 & -0.0239 & 0.1476 \\
\hline $\begin{array}{l}\text { Plant height } \\
(\mathrm{cm})\end{array}$ & -0.0005 & 0.0041 & -0.0063 & 0.0023 & -0.0010 & 0.0059 & 0.0000 & -0.0024 & 0.0271 & -0.0075 & -0.0239 & $\begin{array}{c}- \\
0.0022\end{array}$ \\
\hline $\begin{array}{c}\text { Number of } \\
\text { primary } \\
\text { branches per } \\
\text { plant }\end{array}$ & -0.0003 & 0.0026 & -0.0020 & 0.0002 & -0.0130 & 0.0132 & 0.0000 & -0.0014 & 0.0663 & 0.0015 & 0.0686 & 0.1357 \\
\hline $\begin{array}{c}\text { Number of } \\
\text { pods per plant }\end{array}$ & -0.0010 & 0.0091 & -0.0072 & 0.0004 & -0.0044 & 0.0390 & 0.0000 & -0.0104 & 0.1475 & 0.0007 & 0.2675 & 0.4413 \\
\hline $\begin{array}{c}\text { Pod length } \\
(\mathrm{cm})\end{array}$ & -0.0006 & 0.0068 & -0.0083 & 0.0004 & 0.000 & 0.0027 & -0.0001 & 0.0050 & 0.1009 & 0.0049 & 0.1266 & 0.2377 \\
\hline $\begin{array}{c}\text { Number of } \\
\text { seed per pod }\end{array}$ & 0.0001 & -0.0006 & -0.0003 & $\begin{array}{c}- \\
0.0002\end{array}$ & 0.0005 & -0.0120 & 0.0000 & 0.0338 & 0.3455 & 0.0036 & 0.0398 & 0.4103 \\
\hline $\begin{array}{c}\text { Dry matter } \\
\text { yield per plant } \\
\text { (g) }\end{array}$ & -0.0001 & 0.0026 & -0.0055 & 0.0001 & -0.0015 & 0.0098 & 0.0000 & 0.0200 & 0.5850 & 0.0320 & 0.2048 & 0.8502 \\
\hline $\begin{array}{c}\text { Hundred seed } \\
\text { weight (g) }\end{array}$ & 0.0001 & -0.0004 & -0.0007 & $\begin{array}{c}- \\
0.0003\end{array}$ & -0.0003 & 0.0005 & 0.0000 & 0.0020 & 0.3081 & 0.0607 & 0.2681 & 0.6377 \\
\hline $\begin{array}{l}\text { Harvest index } \\
(\%)\end{array}$ & -0.0006 & 0.0047 & -0.0050 & $\begin{array}{c}- \\
0.0001\end{array}$ & -0.0017 & 0.0196 & 0.0000 & 0.0025 & 0.2244 & 0.0305 & 0.5339 & 0.8082 \\
\hline
\end{tabular}


Table.4 Path analysis at genotypic level for 12 quantitative characters

\begin{tabular}{|c|c|c|c|c|c|c|c|c|c|c|c|c|}
\hline Character & $\begin{array}{l}\text { Days to } \\
\text { first } \\
\text { flowering }\end{array}$ & $\begin{array}{c}\text { Days to } \\
50 \% \\
\text { flowering }\end{array}$ & $\begin{array}{l}\text { Days to } \\
\text { maturity }\end{array}$ & $\begin{array}{c}\text { Plant } \\
\text { height } \\
(\mathrm{cm})\end{array}$ & $\begin{array}{c}\text { Number } \\
\text { of } \\
\text { primary } \\
\text { branches } \\
\text { per plant }\end{array}$ & $\begin{array}{c}\text { Number } \\
\text { of pods } \\
\text { per } \\
\text { plant }\end{array}$ & $\begin{array}{c}\text { Pod } \\
\text { length } \\
\text { (cm) }\end{array}$ & $\begin{array}{l}\text { Number } \\
\text { of seed } \\
\text { per pod }\end{array}$ & $\begin{array}{c}\text { Dry } \\
\text { matter } \\
\text { yield } \\
\text { per } \\
\text { plant } \\
\text { (g) }\end{array}$ & $\begin{array}{l}\text { Hundred } \\
\text { seed } \\
\text { weight } \\
\text { (g) }\end{array}$ & $\begin{array}{c}\text { Harvest } \\
\text { index } \\
(\%)\end{array}$ & $\begin{array}{l}\text { Seed } \\
\text { yield per } \\
\text { plant }(g)\end{array}$ \\
\hline $\begin{array}{l}\text { Days to first } \\
\text { flowering }\end{array}$ & 0.0108 & 0.0712 & -0.0906 & 0.0025 & -0.0055 & -0.0037 & 0.0008 & -0.0006 & 0.0332 & -0.0005 & 0.1633 & 0.1811 \\
\hline $\begin{array}{l}\text { Days to } 50 \% \\
\text { flowering }\end{array}$ & 0.0107 & 0.0721 & -0.0916 & 0.0021 & -0.0043 & -0.0039 & 0.0011 & -0.0004 & 0.0821 & -0.0006 & 0.1740 & 0.2412 \\
\hline Days to maturity & 0.0109 & 0.0731 & -0.0903 & 0.0035 & 0.0006 & -0.0028 & 0.0001 & -0.0002 & 0.1096 & 0.0010 & 0.1196 & 0.2263 \\
\hline Plant height (cm) & 0.0029 & 0.0163 & -0.0335 & 0.0094 & -0.0051 & -0.0015 & 0.0004 & -0.0012 & 0.0402 & -0.0038 & 0.0084 & 0.0324 \\
\hline $\begin{array}{c}\text { Number of } \\
\text { primary branches } \\
\text { per plant }\end{array}$ & 0.0022 & 0.0114 & 0.0019 & 0.0018 & -0.0270 & -0.0039 & 0.0004 & -0.0008 & 0.1046 & 0.0006 & 0.1823 & 0.2734 \\
\hline $\begin{array}{l}\text { Number of pods } \\
\text { per plant }\end{array}$ & 0.0044 & 0.0310 & -0.0279 & 0.0015 & -0.0117 & -0.0091 & 0.0002 & -0.0037 & 0.1628 & 0.0003 & 0.3202 & 0.4680 \\
\hline Pod length (cm) & 0.0025 & 0.0233 & -0.0378 & 0.0011 & -0.0037 & -0.0006 & 0.0033 & 0.0018 & 0.1091 & 0.0022 & 0.1249 & 0.2261 \\
\hline $\begin{array}{c}\text { Number of seed } \\
\text { per pod }\end{array}$ & -0.0006 & -0.0023 & 0.0015 & -0.0010 & 0.0019 & 0.0029 & 0.0005 & 0.0118 & 0.3645 & 0.0014 & 0.0540 & 0.4346 \\
\hline $\begin{array}{c}\text { Dry matter yield } \\
\text { per plant (g) }\end{array}$ & 0.0006 & 0.0102 & -0.0170 & 0.0007 & -0.0049 & -0.0026 & 0.0006 & 0.0074 & 0.5819 & 0.0137 & 0.3361 & 0.9267 \\
\hline $\begin{array}{c}\text { Hundred seed } \\
\text { weight }(\mathrm{g})\end{array}$ & -0.0002 & -0.0018 & -0.0037 & -0.0014 & -0.0007 & -0.0001 & 0.0003 & 0.0007 & 0.3228 & 0.0247 & 0.3147 & 0.6552 \\
\hline $\begin{array}{c}\text { Harvest index } \\
(\%)\end{array}$ & 0.0035 & 0.0251 & -0.0216 & 0.0002 & -0.0099 & -0.0058 & 0.0008 & 0.0013 & 0.3911 & 0.0155 & 0.5001 & 0.9003 \\
\hline
\end{tabular}


Direct effects Path coefficient analysis showed that the characters days to first flowering, days to fifty per cent flowering, plant height, number of pods per plant, number of seeds per pod, first 100 seed weight, pod diameter $(\mathrm{cm})$. These results were conformity with Tyagi et al., (2000) and Singh et al., (2004).

\section{Indirect effects on yield parameters}

Number of seeds per pod had positive indirect effect on dry matter yield per plant, hundred seed weight and harvest index at both phenotypic and genotypic level, while negative indirect effect on days to fifty per cent flowering at both phenotypic and genotypic level. Manggoel (2012) and Kulkarni (1994) reported similar result for indirect effect of dry pod yield per plant and hundred seed weight in cowpea. Dry matter yield per plant had positive indirect effect through hundred seed weight, harvest index, plant height, days to first flowering, days to fifty per cent flowering and number of seeds per pod, while days to maturity and number of pods per plant had negative indirect effect. This result was in confirmation with Kulkarni (1994) and Rangaiah and Mahadevu (2000). Hundred seed weight showed positive indirect effect through number of seeds per pod, dry matter yield per plant, harvest index and hundred seed weight. It showed negative indirect effect on seed yield through days to fifty per cent flowering, days to maturity, plant height and number of primary branches per plant. Pekson and Cengiz (2004) and Manggoel et al., (2012) reported high positive direct effect of hundred seed weight on seed yield per plant. Harvest index showed positive indirect effect on seed yield through number of seeds per pod, dry matter yield per plant and hundred seed weight. It showed negative indirect effect through days to maturity and number of primary branches per plant. This result was similar with the observation of Kulkarni (1994) at genotypic level for seed yield per plant and dry pod yield per plant.
In conclusion, it is to be stated thaton the basis of correlation and path analysis studied, seed yield per plant could be improved through simultaneous selection of number of pods per plant, number of seeds per pod, hundred seed weight and harvest index. It is desirable to give more weightage to these characters in selection program.

\section{References}

Anonymous, 2012.FAO, FAOSTAT. Food and Agriculture Organization of the United Nations, Rome, Italy.

Burton, G. W and E. H. De Vane, 1953. Estimating heritability in tall Fescue (Festuca arundinaceae) from replicated clonal material. Agronomy Journal. 45: 478-481.

Dewey, D. R. and K. H. Lu, 1959. A correlation and path analysis of crested wheat grass and seed production. Agronomy Journal. 51: 315-318.

Johnson, H. W., H. F. Robinson and P. E. Comstock, 1955. Estimate of genetic and environmental variability in Soybeans. Algronomy Journal. 47: 314-318.

Kulkarni, M. P., 1994. Genertc variability, correlation and path analysis in cowpea (Vigna unguiculata (L.)Walp.). A thesis submitted to DBSKKV, Dapoli. (Unpublished).

Malarvizhi, D and Rangasamy, P., 2003. Character association and component analysis in $\mathrm{F}_{2}$ generation of cowpea (Vigna unguiculata L. Walp). Legume Research. 26(4): 264-267

Manggoel, W., M. I. Uguru, O. N. Ndam and M. A. Dasbak, 2012. Genetic variability, correlation and path coefficient analysis of some yield components of ten cowpea (Vigna unguiculata (L.)Walp) assessions. Journal of Plant Breeding and Crop Science, 4 (5): 80-86. 
Oseni, T.O., D.D Lenge and V.R. Pal, 1992. Correlation and path coefficient analysis of yield attributes in diverse lines of cowpea (Vigna unguiculata (L.) Walp). Indian Journal of Agricultural Sciences .62: 365-368.

Panse, V.G and P.V. Sukhatme, 1985. Statistical method for agricultural workers, $4^{\text {th }}$ Edn., ICAR, New Delhi.

Patel, U.V, V.K Parmar, P. B. Patel, and A.V Malviya., 2016. Correlation and path analysis study in cowpea (Vigna unguiculata (L.) Walp.). International Journal of Science, Environment and Technology, Vol. 5: 3897 - 3904.

PeksenErkut and CengizArkit, 2004. Comparison of some cowpea (Vigna unguiculata (L.)Walp.) genotypes from Turkey for seed yield and yield related characters. Journal of Agronomy. 3(2): 137-140.

Rangaiah, S. and P. Mahadevu, 2000.Genetic variability, correlation and path coefficient analysis in cowpea (Vigna unguiculata L. Walp). Madras Agricultural Journal. 86: 7/9, 381-384.

Sapara and Javia. 2014. Correlation and path analysis in vegetable cowpea (Vigna unguiculata. (L). International journal of plant sciences. Vol. 9: 138-141.

Singh, B., A.B. Bagade, D.U. Patel and M.R. Naik. 2004. Correlation and path analysis in cowpea. Indian J. Pulses Res., 17(1): 84-85.

\section{How to cite this article:}

Waghmare, P. D., U. B. Pethe and Sabale, G. R. 2019. Study on Correlation and Path Analysis for Yield and Yield Components in Cowpea [Vigna unguiculata (L.) Walp]. Int.J.Curr.Microbiol.App.Sci. 8(12): 296-303. doi: https://doi.org/10.20546/ijcmas.2019.812.041 